\title{
Estudio longitudinal sobre calidad de vida, craving y ajuste psicológico en pacientes dependientes del alcohol: variaciones en función de los trastornos de la personalidad \\ Longitudinal study on quality of life, craving and psychological adjustment in alcohol-dependent patients: variations depending on the personality disorders
}

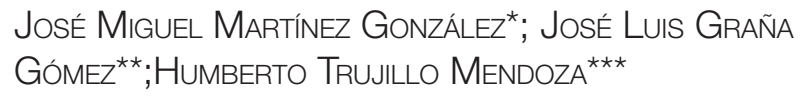

\author{
* Centro Provincial de Drogodependencias de Granada \\ ** Universidad Complutense de Madrid \\ *** Universidad de Granada. \\ Enviar correspondencia a: \\ José Miguel Martínez González \\ Centro Provincial de Drogodependencias de Granada. \\ Hospital de San Juan de Dios. \\ C/ San Juan de Dios, 18001 - Granada \\ jmmgonz@dipgra.es
}

recibido: enero 2011

aceptado: mayo 2011

\section{RESUMEN}

El objetivo de este estudio fue conocer la evolución de variables como la calidad de vida, el craving o el ajuste psicológico a lo largo del tratamiento en una muestra de 65 pacientes con un trastorno por dependencia del alcohol, de los que el 56,3\% presentaba un trastorno de la personalidad (TP). Se realizaron cinco mediciones a lo largo del tratamiento con un intervalo de tres meses entre cada una de ellas. El análisis de tendencias de la variables craving, calidad de vida y ajuste psicológico a lo largo de los cinco momentos en que fueron evaluados los pacientes ponen de manifiesto que el tratamiento cognitivo-conductual influye de distinta manera en cada una de estas variables: estableciéndose una relación cuadrática y cúbica para el craving; para la calidad de vida en sus distintas escalas se da una relación lineal, cuadrática y cúbica según los distintos momentos temporales; y para el ajuste psicológico existe una relación lineal y cuadrática. Al final del tratamiento los pacientes evaluados presentan niveles de calidad de vida significativamente mayores en comparación al inicio del mismo, pero los resultados muestran que aunque los pacientes con TP mejoran su percepción de calidad de vida a los tres meses, es menor a lo largo de todo el tratamiento con respecto al nivel de los pacientes que no presentan TP. También se ha constatado que la calidad de vida y el craving al iniciar el tratamiento predicen el consumo de alcohol durante los tres primeros meses. Se discuten las implicaciones de estos resultados cara a la intervención psicológica con pacientes dependientes del alcohol.

Palabras clave: estudio longitudinal, trastornos de la personalidad, calidad de vida, ajuste psicológico, alcohol, tratamiento cognitivo-conductual, craving.

\section{ABSTRACT}

The goal of this study was to determine the evolution of variables such as quality of life, craving, or psychological adjustment during treatment in a sample of 65 patients with alcohol-dependence disorder, $56.3 \%$ of whom also presented a personality disorder (PD). Five measurements were taken over the course of the treatment, at 3-month intervals. The analysis of tendencies of the variables craving, quality of life, and psychological adjustment assessed at the five assessment points revealed that the cognitive-behavioral treatment influences each one of these variables differently: a quadratic and cubic relation was identified for craving, whereas for quality of life there were linear, quadratic and cubic relationships in its diverse scales depending on the time point in question, and for psychological adjustment there were linear and quadratic relationships. At the end of treatment, the patients assessed presented significantly higher levels of quality of life than at the beginning, but the results showed that, although the patients with PD had better perceived quality of life at three months, it was lower over the entire course of the treatment compared to patients without PD. It was also found that quality of life and craving at the start of the treatment predicted alcohol use during the first three months. The implications of these results are discussed with a view to psychological intervention with alcohol-dependent patients.

Key Words: prospective longitudinal study, personality disorders, quality craving. of life, psychological adjustment, alcohol, cognitive-behavioral treatment, 


\section{INTRODUCCIÓN}

L a calidad de vida ha sido validada a los largo de los últimos años como un indicador del resultado del tratamiento en drogodependencias (Iraurgi, 2002; Morales-Manrique, Castellano-Gómez y Valderrama, 2006; González-Saiz, Lozano y Iraurgi, 2009). En la percepción de la calidad de vida (en adelante CV) se sabe que influyen variables como por ejemplo el carácter de la persona, lo que hace que los trastornos de la personalidad (en adelante TP) (DSMIV-TR, 2002) tengan un peso destacado en el análisis de esta variable (Fassino, Abbate, Delsedime, Rogna, y Boggio, 2004; Karow, Verthein, Krausz y Schäfer, 2008). Tal es así que se ha comprobado que el número de criterios que se cumplen para el diagnóstico del TP correlaciona de forma lineal con el nivel de calidad de vida percibida (Torgersen, 2007).

Los pacientes con TP presentan niveles de CV menores en comparación a la población no clínica (Torgensen, 2007), y sobre todo peores niveles de ajuste social en comparación a los pacientes que solo presentan psicopatología en el Eje I (DSM-IV-TR, 2002). Además debe tenerse en cuenta que existen diferencias en el nivel de CV autopercibido entre los diferentes TP. Mientras que algunos TP como evitación, límite, equizotípico, dependiente, paranoide, esquizoide y antisocial presentan peores niveles, los TP histriónico y narcisista presentan mejores niveles de CV (Daley, Burge y Hammen, 2000; Cramer, Torgersen y Kringlen, 2006; Pedrero, Olivar y Chicharro, 2008).

En un estudio parcial de una investigación más amplia de la que forma parte también este trabajo (Martínez-González, Graña y Trujillo, 2010) se constataron varios aspectos de gran relevancia en el estudio de la $\mathrm{CV}$, el craving y el nivel gravedad de ajuste psicológico (en adelante GAP). El nivel de CV autopercibida al iniciar el tratamiento no depende del tiempo de abstinencia ni la evolución de la drogodependencia. Las medidas de CV correlacionan con el índice de GAP lo que viene a confirmar el hecho de que las personas utilizan su estado afectivo momentáneo como referencia para establecer juicios sobre su felicidad y satisfacción en la vida (Smith y Larson, 2003; Lozano, Rojas, Pérez, González-Saiz, Ballesta, y Izaskum, 2008). Al parecer el bienestar subjetivo refleja estados psicopatológicos (Atkinson, Zibin y Chuang, 1997) y particularmente en pacientes con TP, se constata que la psicopatología en el Eje I explica parte de las dimensiones de la CV (Narud, Mykletun y Dahl, 2005). También se ha visto que la CV autopercibida se ve igualmente influenciada por la presencia de craving, lo que significa que la CV debe evaluarse teniendo en cuenta el índice de GAP y, en drogodependientes, el deseo de consumo. Así, puede decirse que el índice de GAP y el craving son buenos predictores de la calidad de vida autopercibida en drogodependientes. Por otro lado, se observa que el GAP refuerza las creencias sobre el craving, lo que convierte el malestar psicológico en un potente factor de riesgo. Según esos resultados, debe tenerse en cuenta que la pretensión de afrontar el deseo de consumo sin modificar las creencias irracionales que sustentan el craving, no solo parece ineficaz (Martínez-González y Verdejo, 2011), sino que además influye negativamente en la CV autopercibida
(Martínez-González, Graña y Trujillo, 2010). En esta investigación, el craving se conceptualiza como las creencias del paciente sobre el deseo de consumo y el nivel de deseo experimentado en el momento de la evaluación.

Existen pocos estudios sobre la calidad de vida en pacientes dependientes del alcohol a lo largo del tratamiento y tampoco se conoce como afectan los trastornos de la personalidad a la calidad de vida o el índice de GAP en este tipo de pacientes. En el estudio de Pedrero, Olivar y Chicharro (2008) analizaron la CV a lo largo del tratamiento en estos pacientes, pero no obtuvieron diferencias estadísticamente significativas a los tres meses de iniciar el tratamiento. Posiblemente, los cambios en la percepción de la calidad de vida en estos pacientes aparecen de forma lenta y progresiva, lo que puede perjudicar la adherencia al tratamiento, ya que si no observan cambios positivos en su CV con cierta prontitud pueden desmotivarse y como consecuencia abandonar el tratamiento.

Las hipótesis de este trabajo fueron: $1^{\text {a) }}$ la calidad de vida mejorará mientras que el craving y el índice de gravedad de ajuste psicológico disminuirá a lo largo del tratamiento, probablemente por efecto del mismo; y $2^{\text {a) }}$ existirán diferencias en las variables evaluadas en función de la presencia de trastornos de la personalidad. Para probar estas hipótesis se plantearon los siguientes objetivos: (1) conocer la evolución de la percepción de la CV, índice de GAP y craving a lo largo del tratamiento en pacientes alcohólicos; y (2) comprobar si las diferencias existentes en la CV en función de la presencia de TP se mantienen a la largo del tratamiento; y (3) analizar variables, como el craving, índice de GAP y otras con relevancia en el tratamiento, en función de la presencia de TP.

\section{MÉTODO}

\section{Participantes}

Como se ha indicado, este trabajo constituye el estudio de seguimiento de una muestra formada por 65 pacientes que cumplian criterios para la dependencia del alcohol (DSM-IV-TR, 2002) y que estaban en tratamiento en el Centro Provincial de Drogodependencias de Granada (MartínezGonzález, Graña y Trujillo, 2010). La edad media era de 43.26 años, siendo el $70.8 \%(n=46)$ hombres y el $29.2 \%(n=19)$ mujeres. Presentan una media de 11.83 años de evolución de la adicción y en el momento de la evaluación cuentan con una media de 29 días de abstinencia. El 67.2\% de la muestra presenta patología dual. El $23.6 \%$ en el Eje I, de los que el $6.3 \%$ corresponden a trastornos de la ansiedad y el $17.3 \%$ a un trastorno del estado de ánimo. El $56.3 \%(n=36)$ presenta un trastorno de la personalidad, de los que el $25 \%$ (n $=9)$ eran mujeres y el $75 \%(n=27)$ hombres. Los trastornos más frecuentes fueron el obsesivo-compulsivo (32.8\%) y el de dependencia (12.5\%), seguidos del trastorno límite de 
la personalidad (4.7\%), no especificado (3.1\%), histriónico (1.6\%) y por evitación (1.6\%).

Para la selección de la muestra se han tenido en cuenta los siguientes criterios: (a) debían ser personas que no habian estado en tratamiento, al menos, en los dos meses anteriores al inicio de esta investigación; (b) debian estar abstinentes al menos durante dos semanas para poder hacer el diagnóstico dual, y (c) encontrarse en el primer mes de tratamiento para evitar que la evaluación de las variables se viese influida por el efecto de la intervención terapéutica.

En todos los casos se llevó a cabo una intervención psicológica que consistió en la aplicación de un programa de prevención de recaídas con un enfoque cognitivo-conductual (Martínez-González, Graña y Trujillo, 2009) con una duración aproximada de 12 meses.

\section{Instrumentos de evaluación.}

Los instrumentos de evaluación que se utilizaron en esta investigación fueron los siguientes:

a) Para la evaluación de la CV se utilizó el Cuestionario sobre Calidad de Vida: Sastisfacción y Placer (Quality of Life and Satisfaction Questionnaire, P-LES-Q) (Endicott, Harrison y Blumenthal, 1993). Este cuestionario mide de forma sensible la satisfacción y el placer del paciente en su vida cotidiana. Es un instrumento genérico de CV aplicable a todo tipo de pacientes, con independencia de su diagnóstico y tratamiento. Consta de 93 ítems que exploran las siguientes ocho áreas: estado de salud física/actividades $(\alpha=.81)$, estado de ánimo $(\alpha=.92)$, trabajo $(\alpha=.95)$, actividades del hogar $(\alpha=.92)$, tareas de clase $(\alpha=.94)$, actividades de tiempo libre $(\alpha=.88)$, relaciones sociales ( $\alpha=$ $.82)$ y actividades generales $(\alpha=.86)$. La consistencia interna de todas áreas es aceptable. El cuestionario incorpora un apartado resumen para que la persona haga una valoración general de cada área.

b) Para la evaluación de la psicopatología no psicótica se utilizó el Cuestionario General sobre Salud - General Health Questionnarie - GHO-28 (Goldber y Hiller, 1979), el cual establece un indice de gravedad de ajuste psicológico construido a partir de la suma de cuatro subescalas: síntomas somáticos, ansiedad, disfunción social y depresión (González-Saiz et al.,1997).

c) Para la evaluación y diagnóstico de los TP se utilizó la entrevista semiestructurada "Examen Internacional de los Trastornos de la Personalidad (IPDE)" (Loranger, 1995; versión española de López-lbor, Pérez Urdániz y Rubio, 1996).

d) La evaluación del Eje I se llevó a cabo a través de la entrevista clínica, atendiendo a criterios DSM (DSMIV-TR, 2002).

e) A través de un cuestionario de elaboración propia, se ha evaluado la percepción de la necesidad de ayuda de profesionales y el craving con respuestas tipo Likert, de cinco valores que van desde ningún grado de identificación con las creencias a muchísimo. El craving viene dado por el valor de la media de ocho ítems y para conocer la percepción de necesidad de ayuda de los profesionales se utilizó el ítem "necesito la ayuda de los profesionales". Los ítems de este cuestionario que se refieren al craving presentan un valor Alfa de Cronbach de .89, lo que indica una alta consistencia interna.

\section{Procedimiento}

Se realizaron 5 mediciones a lo largo del tratamiento. La primera durante el primer mes en las condiciones que se han descrito anteriormente. La segunda a los tres meses de iniciar el tratamiento con un margen de una semana para completar los cuestionarios. Si el paciente por alguna razón no completaba los cuestionarios en la semana designada quedaba excluido del estudio. De este modo la tercera medición se hizo a los seis meses, la cuarta a los nueve y la quinta a los doce meses desde que el paciente inicia el tratamiento.

Se solicitó a todos los participantes el consentimiento informado para la utilización de los datos en esta investigación.

\section{Diseño y análisis estadístico}

Se empleó una metodología cuasi-experimental, consistente en la utilización de distintos diseños unifactoriales entre grupos e intrasujetos. Se realizaron análisis de tendencias y distintos contrastes sobre diferencias de medias mediante el estadístico t - Student. También se realizaron correlaciones bivariadas y, además, análisis de regresión simple y múltiple, con el fin de conocer la capacidad predictiva de algunas de las variables. El análisis se realizó con el programa SPSS .15.0.

\section{RESULTADOS}

\section{Finalización del tratamiento}

En el análisis de la finalización del tratamiento del conjunto de la muestra, de acuerdo con la tipificación empleada por el Sistema de Información del Plan Andaluz sobre Drogodependencias y Adicciones de la Junta de Andalucía, se observa que el $36,4 \%(n=20)$ abandonó el tratamiento -pacientes que dejan de acudir a las sesiones sin haber consolidado la abstinencia-, finalizó con alta voluntaria el 18,2\% ( $n=10)$-pacientes que dejan de acudir al centro cuando han superado los dos meses de abstinencia y han instaurado un cambio de estilo de vida favorecedor de la abstinencia - y 
alta terapéutica el $45,5 \%(n=25)$-que son los pacientes que han permanecido en tratamiento al menos doce meses habiendo consolidado la abstinencia y alcanzado los objetivos terapéuticos propuestos al inicio del tratamiento-. Los que abandonan el tratamiento lo hacen de media a los 5,13 meses y el $21,5 \%$ lo hace a lo largo de los tres primeros meses. Del grupo de pacientes con TP, abandonó el tratamiento el $34,5 \%$, fue alta voluntaria el $13,8 \%$ y alta terapéutica el $51,7 \%$.

\section{El análisis longitudinal de las variables estudiadas}

Los análisis de tendencias muestran lo siguiente: para la variable dependiente "craving" muestra que son significativos los componentes cuadrático $\left(F_{1,6}=7,413 ; p=0,035\right)$, cúbico $\left(F_{1,6}=6,690 ; p=0,041\right) y$, tangencialmente, el lineal $\left(F_{1,6}=5,132 ; p=0,064\right)$; para la variable dependiente escala general del cuestionario de CV muestra que son significativos los componentes lineal $\left(F_{1,8}=8,116 ; p=0,022\right)$, cua- drático $\left(F_{1,8}=14,898 ; p=0,006\right)$ y cúbico $\left(F_{1,8}=6,088 ; p\right.$ $=0,040)$; en el caso de la variable dependiente de la escala de estado de ánimo del cuestionario de CV son significativos los componentes lineal $\left(F_{1,8}=6,476 ; p=0,034\right)$ y cuadrático $\left(F_{1,8}=13,032 ; p=0,007\right)$; en relación a la escala de relaciones sociales de la variable $\mathrm{CV}$ son significativos los componentes lineal $\left(F_{1,7}=11,613 ; p=0,011\right)$ y cuadrático $\left(F_{1,7}=11,148 ; p=\right.$ $0,012) ;$ para la variable escala estado físico del cuestionario de CV muestra que los componentes significativos son el de orden $4\left(F_{1,8}=32,985 ; p=0,000\right)$ y lineal $\left(F_{1,8}=5,334 ; p=\right.$ $0,05)$, lo que significa que el estado físico va subiendo pero en dientes de sierra; y en el caso de la variable GAP muestra que los componentes significativos son el lineal $\left(\mathrm{F}_{1,8}=\right.$ $17.468 ; p=0,003)$ y el cuadrático $\left(F_{1,8}=12,586 ; p=0,008\right)$ (tabla 1).

El análisis de tendencias muestra que la CV de los pacientes en los cincos momentos evaluados difieren entre sí de forma estadisticamente significativa. Al parecer, la influencia del tratamiento difiere según cada momento (gráfica 1).

Tabla 1. Medias y desviaciones típicas de las diferencias variables en las cinco evaluaciones.

\begin{tabular}{|c|c|c|c|c|c|c|c|c|c|}
\hline \multirow[t]{2}{*}{ Evaluación } & \multicolumn{3}{|c|}{$\begin{array}{c}\text { CV General } \\
\text { Grupo de persona con TP }\end{array}$} & \multicolumn{3}{|c|}{$\begin{array}{c}\text { CV General } \\
\text { Grupo de personas sin TP }\end{array}$} & \multicolumn{3}{|c|}{$\begin{array}{l}\text { CV General } \\
\text { muestra }\end{array}$} \\
\hline & $M$ & DT & $\mathrm{N}$ & M & DT & $\mathrm{N}$ & $M$ & DT & $\mathrm{N}$ \\
\hline Primera & 51,83 & 4,491 & 36 & 54,5 & 0,707 & 29 & 51,6 & 11,293 & 65 \\
\hline Segunda & 60,67 & 7,992 & 19 & 62 & 1,414 & 12 & 58,1 & 14,494 & 31 \\
\hline Tercera & 65,17 & 7,468 & 14 & 65,5 & 2,121 & 7 & 64,29 & 8,235 & 21 \\
\hline Cuarta & 57,33 & 12,894 & 11 & 66,5 & 3,536 & 6 & 59,88 & 10,582 & 17 \\
\hline Quinta & 61,5 & 11,640 & 7 & 62 & 2,828 & 4 & 63 & 9,263 & 11 \\
\hline \multirow[t]{2}{*}{ Evaluación } & \multicolumn{3}{|c|}{$\begin{array}{c}\text { GHQ-28 (GAP) } \\
\text { Grupo de persona con TP }\end{array}$} & \multicolumn{3}{|c|}{$\begin{array}{c}\text { GHQ-28 (GAP) } \\
\text { Grupo de personas sin TP }\end{array}$} & \multicolumn{3}{|c|}{$\begin{array}{c}\text { GHQ-28 (GAP) } \\
\text { muestra }\end{array}$} \\
\hline & $M$ & DT & $\mathrm{N}$ & M & DT & $\mathrm{N}$ & $M$ & DT & $\mathrm{N}$ \\
\hline Primera & 11,71 & 11,71 & 32 & 11 & 15,55 & 26 & 11,88 & 8,868 & 58 \\
\hline Segunda & 5,43 & 1,414 & 20 & 1 & 5,255 & 12 & 5,06 & 7,440 & 32 \\
\hline Tercera & 1,9 & 0,787 & 14 & & 6,807 & 6 & 2,5 & 5,375 & 20 \\
\hline Cuarta & 2,6 & 12,894 & 11 & 6,25 & 2,887 & 6 & 3,06 & 6,666 & 17 \\
\hline Quinta & 3,5 & 4,237 & 7 & 2,43 & 2,121 & 4 & 2,27 & 3,495 & 11 \\
\hline \multirow[t]{2}{*}{ Evaluación } & \multicolumn{3}{|c|}{$\begin{array}{c}\text { Craving } \\
\text { Grupo de personas con TP }\end{array}$} & \multicolumn{3}{|c|}{$\begin{array}{c}\text { Craving } \\
\text { Grupo de personas sin TP }\end{array}$} & \multicolumn{3}{|c|}{$\begin{array}{l}\text { Craving } \\
\text { muestra }\end{array}$} \\
\hline & $M$ & DT & $\mathrm{N}$ & $M$ & DT & $\mathrm{N}$ & M & DT & $\mathrm{N}$ \\
\hline Primera & 0,75 & 1.254 & 35 & 1,38 & 0,177 & 28 & 0,96 & 0,983 & 63 \\
\hline Segunda & 0,09 & 0,09 & 20 & 0,25 & 0,354 & 13 & 0,49 & 0,739 & 33 \\
\hline Tercera & 0,09 & 0,188 & 14 & 0,08 & 0,168 & 6 & 0,28 & 0,535 & 20 \\
\hline Cuarta & 0,19 & 0,422 & 10 & 0,25 & 0,354 & 6 & 0,16 & 0,349 & 16 \\
\hline Quinta & 0,08 & 0,179 & 6 & 0,75 & 0,354 & 4 & 0,19 & 0,341 & 10 \\
\hline
\end{tabular}




\section{Evolución de las diferentes variables a lo largo del estudio.}

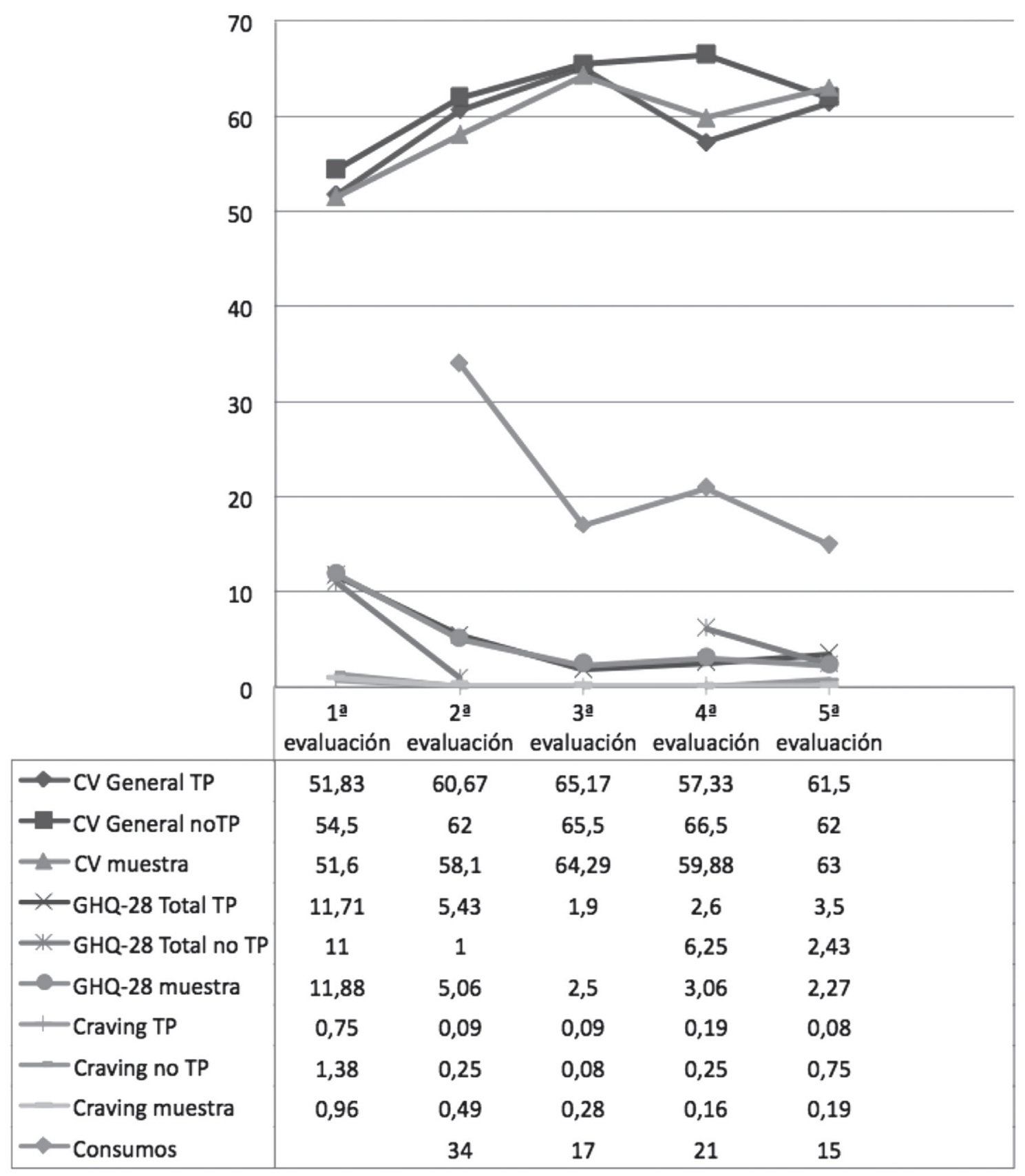

La evolución de la calidad de vida y el nivel de gravedad de ajuste psicológico

Para conocer el cambio en CV y GAP a lo largo del tratamiento se compararon las puntuaciones en CV y GAP de los pacientes al iniciar el tratamiento y a los tres y doce meses, habiendo obtenido en ambas diferencias estadísticamente significativas. A los tres meses la CV aumenta significativamente $(t=-3,161 ; p=0,005)$, al igual que a los doce meses, observando que la media de las puntuaciones es mayor a los doce meses de tratamiento $(t=-3,417 ; p=0,007)$. Por el contrario, con el ajuste psicológico a los doce meses disminuye $(t=4,499 ; p=0,001)$ (tabla 2$)$ (gráfica 1$)$.

\section{El craving a los tres meses en tratamiento}

Se han obtenido diferencias estadísticamente significativas entre las puntuaciones de craving al inicio de tratamiento y a los tres meses. Esto es, el craving experimenta una disminución estadísticamente significativa en los tres primeros meses de tratamiento porque la puntuación de craving disminuye significativamente $(t=3,655 ; p=0,001)$ (tabla 2$)$. 


\section{La relación craving y gravedad de ajuste psicológico} a lo largo del tratamiento

Se han realizado diversas regresiones lineales para conocer la capacidad predictiva del craving sobre la GAP en diferentes momentos del tratamiento. Se observa que al iniciar el tratamiento (Beta $=0,440, p=0,001$ ) y a los tres meses de tratamiento (Beta $=0,463 ; p=0,008$ ) el craving predice en sentido positivo el nivel de GAP. En ambos casos se incrementa la gravedad del ajuste psicológico en la medida en que los pacientes se identifican más con las creencias relacionadas con el craving. No sucede lo mismo a los seis, nueve y doce meses de tratamiento, lo que indica que el craving experimentado en fases más avanzadas del tratamiento no causa el mismo malestar que el descrito durante los tres primeros meses.

\section{La calidad de vida, el craving y la gravedad de ajuste psicológico como predictores de los consumos a lo largo de los tres primeros meses de tratamiento}

La correlación de Pearson entre la medida inicial de CV y el número de consumos que realizan los pacientes durante los tres primeros meses es negativa y estadísticamente significativa $(r=-0,334 ; p=0,031)$.

El análisis de regresión indica que la calidad de vida, en su apartado de valoración general, predice el número de consumos a lo largo de los tres primeros meses (Beta $=-0,334$; $p=0,031)$, de modo que cuanto mayor sea la calidad de vida autopercibida al iniciar el tratamiento menos probable es que consuma el paciente en ese periodo. Esta misma relación se ha encontrado con el craving. La correlación entre el valor de la media de los ítems referentes al craving y el número de consumos es positiva y estadísticamente significativa $(r$ $=0,343 ; p=0,028)$. Se ha analizado también la capacidad predictiva de estos ítems relacionados con el craving sobre los consumos a lo largo de los tres primeros meses, comprobando que predicen el número de consumos a lo largo de los tres primeros meses (Beta $=0,353 ; p=0,005)$. Por el contrario, el la GAP al iniciar el tratamiento no tiene la capacidad de predecir el número de consumos a lo largo de los tres primeros meses de tratamiento.

\section{Impacto del consumo a lo largo de los tres primeros meses sobre el craving.}

Se ha querido indagar en el modo en que puede afectar el consumo a lo largo de los tres primeros meses. Si se evalúa el craving descrito por los pacientes a los tres meses se observa que el craving es mayor en el grupo de pacientes que habian consumido en alguna ocasión ( $t=3,684 ; p=0,001)$, lo que muestra el impacto de los consumos en esta variable (tabla 2).

\section{Pacientes con trastornos de la personalidad}

\section{La adherencia al tratamiento}

Para indagar en la adherencia al iniciar el tratamiento se ha preguntado a los pacientes sobre el grado en el que creen necesitar la ayuda de un profesional para superar su drogadicción. Se han comparado los grupos en función de la presencia de TP y se ha comprobado que los pacientes que no presentan TP se diferencian de forma estadisticamente significativa de los que sí presentan esta psicopatología. Los pacientes sin TP manifiestan más necesidad de ayuda de los profesionales $(t=-2,533 ; p=0,014)$ (tabla 2$)$.

La calidad de vida a lo largo del tratamiento en pacientes con trastornos de la personalidad

Las diferencias en CV al iniciar el tratamiento en función de la presencia de TP permanecen a lo largo del tratamiento, aunque dejan de ser estadísticamente significativas a los tres meses (gráfica 1). Al comparar las puntuaciones de CV de los pacientes con trastornos de la personalidad al iniciar el tratamiento y a los tres meses se observa que la calidad de vida a los tres meses es significativamente mayor $(\mathrm{t}=$ $-3,161 ; p=0,005$ ) (tabla 2).

El número de consumos, gravedad de ajuste psicológico y calidad de vida en función de los trastornos de la personalidad

No se encuentran diferencias estadísticamente significativas con respecto al número de consumos a lo largo de los tres primeros meses en función de la presencia de trastornos de la personalidad. También se ha analizado mediante una regresión logística la capacidad predictiva de los TP sobre los consumos durante los tres primeros meses, confirmando que la presencia de TP no predice los consumos.

En la gráfica 1 puede observarse la evolución de todas las variables estudiadas a lo largo de las evaluaciones en el grupo de pacientes con TP.

Si comparamos exclusivamente las puntuaciones de los pacientes con TP en craving, calidad de vida y GAP al inicio de tratamiento y a los tres meses, vemos que a los tres meses de tratamiento presentan una CV significativamente mayor $(t=-3,161 ; p=0,005)$ y menor índice de GAP $(t=3,663 ; p$ $=0,002)$. En relación a la GAP, se observa que los pacientes también mejoran significativamente en las escalas de ansiedad, disfunción social y dependencia. No se observan diferencias estadísticamente significativas en craving a diferencia de lo que sucede con la totalidad de la muestra que experimenta una disminución significativa del craving, lo que implica que en pacientes con TP tarda más tiempo en disminuir (tabla 2).

Se han realizado diferentes regresiones para conocer la capacidad predictiva de variables como el craving, la calidad de vida y la GAP sobre los consumos a lo largo de los tres primeros meses de tratamiento en pacientes con trastornos de la personalidad. Se ha visto que ni el craving ni el índice de GAP tienen capacidad para predecir los consumos, mientras que la calidad de vida, en su escala de valoración general, sí la tiene (Beta $=-0,436 ; p=0,029)$.

\section{DISCUSIÓN}

Este trabajo nos ha permitido confirmar nuestras hipótesis de partida a la vez que profundizar en una serie de aspec- 
Tabla 2. Análisis de "t-Student" de las diferentes variables.

\begin{tabular}{|c|c|c|c|c|c|c|}
\hline & Grupo de comparación & Media & $\begin{array}{l}\text { Desviación } \\
\text { típica }\end{array}$ & $\mathrm{t}$ & gl & $p$ \\
\hline \multirow[t]{2}{*}{ Calidad de vida } & CV al iniciar el tratamiento. & 50,84 & 10,84 & $-3,161$ & 30 & 0,005 \\
\hline & CV a los tres meses de iniciar tratamiento & 57,68 & 10,26 & & & \\
\hline \multirow[t]{2}{*}{ Calidad de vida } & CV al inicio de tratamiento. & 53,00 & 4,27 & $-3,417$ & 10 & 0,007 \\
\hline & A los doce meses de tratamiento & 63,00 & 9,26 & & & \\
\hline \multirow{2}{*}{$\begin{array}{l}\text { Gravedad de ajuste } \\
\text { psicológico GHQ-28 }\end{array}$} & GHO-28 inicial. & 11,64 & 6,47 & 4,499 & 10 & 0,001 \\
\hline & GHO-28 a los 12 meses de tratamiento & 2,27 & 3,49 & & & \\
\hline \multirow[t]{2}{*}{ Craving } & Craving al inicio del tratamiento & 0,95 & 0,94 & 3,655 & 31 & 0,001 \\
\hline & Craving a los tres meses & 0,50 & 0,74 & & & \\
\hline \multirow{2}{*}{$\begin{array}{l}\text { Craving a los tres meses } \\
\text { de tratamiento }\end{array}$} & Ha consumido durante los tres primeros meses & 1,20 & 0,450 & 3,684 & 31 & 0,001 \\
\hline & No ha consumido durante los tres primeros meses & 0,26 & 1,01 & & & \\
\hline \multirow{2}{*}{$\begin{array}{l}\text { Al iniciar el tratamiento: } \\
\text { "Necesito la ayuda de los } \\
\text { profesionales". }\end{array}$} & Presentan trastorno de la personalidad & 3,17 & 0,891 & $-2,533$ & 60 & 0,014 \\
\hline & No presentan un trastorno de la personalidad & 3.67 & .555 & & & \\
\hline \multirow[t]{2}{*}{ CV en pacientes con TP. } & Al iniciar el tratamiento & 50,84 & 10,84 & $-3,161$ & 18 & 0,005 \\
\hline & A los tres meses de iniciar el tratamiento & 57,68 & 10,26 & & & \\
\hline \multirow{2}{*}{$\begin{array}{l}\text { GHQ-28 en pacientes } \\
\text { con TP }\end{array}$} & Al iniciar el tratamiento & 12,00 & 7,97 & 3,663 & 16 & 0,002 \\
\hline & A los tres meses de iniciar el tratamiento. & 4,76 & 7,45 & & & \\
\hline
\end{tabular}

tos relevantes para mejorar la intervención psicológica con pacientes alcohólicos que presentan trastornos de la personalidad. Se constata que la CV mejora a la vez que disminuye el índice de GAP y el craving a lo largo del tratamiento. También se evidencia que los pacientes con TP presentan niveles de CV menores, en comparación a los que no presentan esta psicopatología, a lo largo de todo el tratamiento. Por otro lado, se observan algunas diferencias en las variables evaluadas en función de la presencia de TP.

Se podría decir que cuando inician tratamiento los pacientes con TP manifiestan menos necesidad de ayuda por parte de los profesionales en comparación a los que no presentan esta psicopatología. Esto es un indicador del impacto de la egosintonía en la construcción de la relación terapéutica y como consecuencia en la adherencia al tratamiento. Esto es, el hecho de que el paciente no sea consciente de su trastorno le impide solicitar ayuda en términos sintomáticos, a la vez que le lleva a interpretar erróneamente los problemas que le sobrevienen en la interacción con otras personas. La dificultad que queda al descubierto muestra la importancia que tiene utilizar estrategias específicas según cada perfil de personalidad para la facilitación de la adherencia al tratamiento (Martínez-González y Trujillo, 2003).

En relación a la finalización del tratamiento se encuentran tasas de alta voluntaria y terapéutica similares a las obtenidas en un estudio anterior (Martínez-González, Graña y Trujillo, 2009). El porcentaje de alta en el grupo de pacientes con TP muestra que el modelo de intervención podría ser efectivo para el tratamiento de este tipo de patología dual. Por otro lado, la calidad de vida como indicador de la efectividad del tratamiento muestra que los pacientes que completaron el tratamiento presentan mejor calidad de vida y mejor índice de GAP en el momento de la finalización del mismo. Estos resultados muestran que el tratamiento produce cambios significativos en estas variables, que como se sabe son un referente en la evaluación de la eficacia del tratamiento en drogodependencias.

En relación al craving también se ha visto que disminuye significativamente después de los tres primeros meses de tratamiento, permitiendo a los pacientes experimentar una mejora que sin duda favorece la adherencia. Sin embargo, esta disminución no es estadísticamente significativa en el grupo de pacientes que presentan TP, lo que indica que los pacientes con esta psicopatología se enfrentan durante más tiempo al craving toda vez que no decrece tan rápidamente. El craving se convierte en un objetivo terapéutico de primera magnitud en los primeros meses de tratamiento, de modo que si se quiere mejorar la adherencia al tratamiento, particularmente en pacientes con TP, se debe evaluar e intervenir sobre el craving tal y como lo hemos conceptualizado en éste estudio. 
Coincidiendo con Weiss, Griffin y Hufford (1995); citados en López y Becoña, (2006) se ha comprobado, en esta ocasión con alcohólicos, que el craving experimentado al iniciar el tratamiento predice el número de consumos a lo largo de los tres primeros meses, pero no a los seis, nueve y doce meses. La relación entre las variables craving y el número de consumos demuestra la vinculación de estas creencias sobre el craving y la probabilidad de consumir alcohol. También se ha visto que el nivel de craving predice la GAP a los tres y seis meses de tratamiento, pero no en etapas más avanzadas. El hecho de que el craving, después de un periodo de tratamiento deje de provocar niveles elevados de malestar prueba que a través de la intervención terapéutica se consigue entrenar al paciente para afrontar el malestar derivado del deseo de consumo. Los pacientes que permanecen más de nueve meses en tratamiento parecen, por tanto, tener más capacidad para neutralizar el malestar derivado del craving, lo que es un indicador positivo de los resultados del tratamiento.

En relación al impacto del consumo sobre el nivel de craving se observa que es significativamente mayor a los tres meses de tratamiento para el grupo de pacientes que habían consumido a lo largo de ese tiempo, demostrando que las creencias vinculadas al craving permanecen activas cierto tiempo tras un consumo, reavivándose de este modo el deseo.

Al iniciar el tratamiento se ha visto que los niveles de $\mathrm{CV}$ percibidos en pacientes con TP son significativamente menores que los que no presentan esta psicopatología (Narud, Mykletun y Dahl, 2005). A diferencia de los resultados del estudio de Pedrero, Olivar y Chicharro (2008) en nuestro estudio se observa que la CV de los pacientes con TP es significativamente mayor a los tres meses, lo que nos hace pensar que tratándose de un trastorno con tendencia a la estabilidad, el paciente drogodependiente con TP puede experimentar mejoras derivadas del tratamiento en un tiempo relativamente corto. Probablemente el impacto del carácter sobre la percepción de la CV disminuye en alguna medida a partir del tercer mes de tratamiento. Nos encontramos ante la evidencia de que es posible introducir pequeños cambios en los hábitos de una persona con TP en un periodo relativamente corto de tiempo aunque los indicadores de mejoría fluctúen a lo largo del tratamiento, lo que podría tener implicaciones considerables en la adherencia al mismo. Estos resultados muestran que la CV de los pacientes con TP mejora a lo largo del tratamiento (Fernández-Hermida, 2008).

Se ha comprobado que la presencia de TP no predice por sí sola los consumos en los tres primeros meses. Sin embargo, un nivel de CV alto se asocia a menos consumos durante los tres primeros meses de tratamiento, lo que demuestra el impacto que puede tener la CV autopercibida al iniciar el tratamiento. Según esto, los pacientes con TP que presenten niveles más bajos de CV deberán someterse a un seguimiento más estricto para contrarrestar este mayor riesgo de recaída. La CV autopercibida y el craving predicen el número de consumos en los primeros meses, convirtiéndose en variables claramente relevantes para el diseño de la intervención en fases iniciales de la intervención psicológica.

\section{CONCLUSIONES}

Del estudio se desprende que el tratamiento es efectivo para el conjunto de la muestra y en particular para los pacientes con $\mathrm{TP}$, dado que produce una mejoría significativa en la CV, así como una reducción del craving experimentado por el paciente y de los niveles de GAP. A los doce meses de tratamiento la CV autopercibida es significativamente mayor en comparación a la que describen los pacientes al iniciar el tratamiento. Por tanto puede decirse que los pacientes con TP se benefician del tratamiento como el resto de los pacientes.

La sola presencia de TP no predice el número de consumo a lo largo de los tres primeros meses de la intervención psicológica de modo que las variables que predicen estos consumos en este periodo son la CV y el craving.

El análisis de tendencias de las variables -craving, CV y GAP- a lo largo de las cinco mediciones del estudio nos revela que el tratamiento podría estar influyendo en estas variables de diferente modo a lo largo del mismo. La CV, el craving y el GAP se relacionan entre sí, por lo que el tratamiento puede influir de diferente modo dependiendo, además de otras, del estado de cada una de estas variables. Por ejemplo, el craving y GAP influyen en el nivel de CV autopercibida tal y como se publicó en un trabajo previo (MartínezGonzález, Graña y Trujillo, 2010) pero además se ha podido ver que estas variables mejoran a lo largo del tratamiento. Todo esto muestra que las estrategias terapéuticas no solo deben guiarse por la personalidad del paciente, adecuarse a las recaídas o consumos puntuales de droga, o a las diferencias individuales que introduce la patología dual, sino que deben tener en cuenta la influencia multifactorial de estas variables en el paciente que al parecer tienen un impacto diferente en distintos momentos del tratamiento.

\section{REFERENCIAS}

Atkinson, M., Zibin, S. y Chuang, H. (1997). Characterizing Quality of life among patients with chronic mental illness: A critical examination of the self-report methodology. American Journal of Psychiatry, 105, 99-105.

Cramer, V., Torgersen, S., y Kringlen, E. (2006). Personality disorders and quality of life. A population study. Comprehensive Psychiatry, 47, 178-184.

Daley, S., Burge, D. y Hammen, C. (2000). Borderline personality disorder symptoms as predictors of 4-year romantic relationship dysfunction in young women: addressing issues of specificity. Journal of Abnormal Psychology, 109, 451-460.

DSM-IV-TR (2002). Manual diagnóstico y estadistico de los trastornos mentales, IV Texto Revisado. Barcelona: Masson.

Endicott, J., Harrison, N.W. y Blumenthal, W. 1993. Quality of Life Enjoyment and Satisfaction Questionnaire: A new Measure. Psychopharmacol Bull, 29, 321-326. 
Fassino, S., Abbate, G., Delsedime, N., Rogna, L., y Boggio, S. (2004). Quality of life and personality disorders in heroin abusers. Drug and Alcohol Dependence, 76, 73-80.

Fernández-Hermida, J.J. (coord) (2008). Opiáceos. Guías Clínicas Socidrogalcohol basadas en la evidencia cientifica. Barcelona. Socidrogalcohol.

González-Saiz, F.M., Carulla, S., Martínez J.M., López, A., Ruiz, J. y Guerra, D. (1997). Indicador del tratamiento de la adicción a opiáceos. Cádiz: Servicio de Publicaciones de la Universidad de Cádiz.

González-Saiz, F., Lozano, O. y Iraurgi, I. (2009). Measuring the impact of psychoactive substance on health-related quality of life: an update. Current Drug Abuse Reviews, 2, 5-10.

Golberg, D.P. y Hiller, V.F. (1979). A scaled version of the General Health Questionaire. Windsor: NFER Publishing Company.

Iraurgi, I., (2002). Instrumentos de evaluación de la calidad de vida relacionada con la salud en toxicomanías. En I Iraurgi y F González (edit). Instrumentos de evaluación en drogodependencias (pp. 481-511). Barcelona: Aula Médica.

Karow, A., Verthein, U., Krausz, M., y Schäfer, I. (2008). Association of personality disorders, family conflicts and treatment with quality of life in opiate addiction. European Addiction Research, $14,38-46$.

López, A. y Becoña, E. (2006). El craving en personas dependientes de cocaina. Anales de psicología, 22, 205-211.

López-lbor, J., Pérez, A., y Rubio, V. (1996). Examen Internacional de los Trastornos de la Personalidad; Módulo DSM-IV.Versión Española. Madrid. Organización Mundial de la Salud.

Lozano, O., Rojas, A., Pérez, C., González-Saiz, F., Ballesta, R., y Izaskum, B. (2008). Evidencias de validez del test para la evaluación de la calidad de vida en adictos a sustancias psicoactivas a partir del modelo biaxial de la adicción. Psicothema, 20, 317-323.
Martínez-González, J.M. y Trujillo, H.M (2003). Tratamiento del drogodependiente con trastornos de la personalidad. Madrid: Biblioteca Nueva.

Martínez-González, J.M., Graña, J.L. y Trujillo, H.M. (2009). Influencia de los trastornos de la personalidad y patrones de consumo en la eficacia de un programa de prevención de recaídas para el tratamiento del alcoholismo. Adicciones, 2, 105-112.

Martínez-González, J.M., Graña, J.L. y Trujillo, H.M. (2010). La calidad de vida en alcohólicos con trastornos de la personalidad: relación con el ajuste psicológico y el craving. Psicothema, 2, 562-567.

Martinez-González, J.M. y Verdejo, A. (2011). Creencias básicas adictivas y craving. Adicciones, 1, 339-352

Morales-Manrique, C., Castellano-Gómez, JC y Valderrama, R. (2006). Medición de la calidad de vida e importancia de la atención a las necesidades autopercibidas en pacientes drogodependientes. Trastornos adictivos, 8, 212-221.

Narud, K., Mykletun, A. y Dahl, A. (2005). Quality of life in patients with personality disorders seen at an ordinary psychiatric outpatient clinic. BMC Psychiatry, 5, 5. En línea [http://www. biomedcentral.com/1471-244X/5/10].

Pedrero, E., Olivar, A. y Chicharro, J. (2008). Cuestionario CAD-4: una medida biopsicosocial de la calidad de vida autopercibida en pacientes drogodependientes. Trastornos Adictivos, 10, 17-31.

Smith, K. y Larson, M. (2003). Quality of life assessments by adult substance abusers receiving publicly funded treatment in Massachusetts. American Journal Alcohol Abuse, 29, 323-335.

Torgersen, S. (2007). Epidemiología. En J.M. Oldham, A E Skodol y DS Bender, Tratado de los trastornos de la personalidad, pp-131143. Barcelona: Elsevier Masson. 
